\title{
Determinantes psicossociais do comportamento sedentário dos idosos: estudo qualitativo
}

\section{Determinantes psicosociales del comportamiento sedentario de adultos mayores: estudio cualitativo \\ Psychosocial determinants of sedentary behavior among older adults: qualitative study}

\author{
Ramalho, André ${ }^{1}$, Petrica, João ${ }^{1}$, Rosado, António ${ }^{2}$ \\ ${ }^{1}$ Sport, Health \& Exercise Research Unit (SHERU) - Instituto Politécnico de Castelo Branco $;{ }^{2}$ Faculdade \\ de Motricidade Humana - Universidade de Lisboa
}

\begin{abstract}
RESUMO
Nos últimos anos verificou-se um aumento progressivo acerca da investigação sobre o comportamento sedentário. No entanto, as evidências empíricas ainda são escassas relativamente aos determinantes psicossociais do tempo sentado dos idosos. Assim, o objetivo do estudo foi identificar e compreender os fatores psicossociais que determinavam os diferentes comportamentos sedentários dos idosos. Participaram no estudo 20 idosos $(M=68$ anos; $D P=1.6$ anos) selecionados intencionalmente. Os dados foram recolhidos através de um guião de entrevista semiestruturada e a análise dos dados seguiu um processo indutivo. Os resultados indicaram os seguintes determinantes psicossociais do comportamento sedentário dos idosos: hábito, comportamentos compensatórios, representação positiva dos comportamentos sedentários e o suporte social. Parece que os comportamentos sedentários dos idosos podem ser determinados por processos automáticos e intencionais. Os idosos também podem utilizar estratégias cognitivas com a intenção de compensar o tempo sentado através de comportamentos ativos e o suporte social dos idosos pode contribuir para a promoção do tempo sedentário ou para a interrupção do mesmo. Desta forma, o entendimento dos fatores psicossociais constitui-se numa importante contribuição para a explicação do tempo sedentário dos idosos.
\end{abstract}

Palavras chave: sedentarismo, envelhecimento, fatores epidemiológicos.

\section{RESUMEN}

En los últimos años se ha producido un aumento progresivo en la investigación sobre el comportamiento sedentario. Sin embargo, las evidencias empíricas todavía son escasas respecto a los determinantes psicosociales del tiempo sentado de adultos mayores. Así, el objetivo del estudio fue identificar y comprender los factores psicosociales que determinaban los diferentes comportamientos sedentarios de los ancianos. Participaron en el estudio 20 ancianos $(M$ $=68$ años, $S D=1,6$ años) seleccionados intencionalmente. Los datos fueron recogidos a través de un guión de entrevista semiestructurado constituido por un conjunto evolutivo de cuestiones y el análisis de los datos siguió un proceso inductivo. Los resultados indicaron los siguientes determinantes psicosociales del comportamiento sedentario de los ancianos: fuerza del hábito, comportamientos compensatorios, representación positiva de los comportamientos sedentarios y el soporte social. Parece que los comportamientos sedentarios de los adultos mayores pueden ser determinados por procesos automáticos e intencionales. Los adultos mayores también pueden utilizar

Correspondence to: André Ramalho. Rua Prof. Dr. Faria de Vasconcelos

6000-266 Castelo Branco, Portugal.

andre.ramalho@ipcb.pt 


\section{Ramalho, A., Petrica, J., Rosado, A.}

estrategias cognitivas con la intención de compensar el tiempo sentado a través de comportamientos activos y el soporte social de los adultos mayores puede contribuir a la promoción del tiempo sedentario o para la interrupción del mismo. De esta forma, el entendimiento de los factores psicosociales se constituye en una importante contribución a la explicación del tiempo sedentario de los adultos mayores.

Palabras clave: sedentarismo, envejecimiento, factores epidemiológicos.

\section{ABSTRACT}

In recent years there has been a progressive research about sedentary behavior. However, the empirical evidence is still scarce regarding the psychosocial determinants of the siting time in older adults. Thus, the main purpose of the study was to identify and understand the psychosocial factors that determined the different sedentary behaviors of older adults. A total of 20 elderly persons, aged between 65 and 75 years ( $M=68$ years; $S D=1.6$ years) participated in this study. The participants were selected intentionally and data was collected through a semi-structured interview guide and data analysis followed an inductive process. Results indicated that following psychosocial determinants of the sedentary behavior in older adults: strength of habit, compensatory behaviors, positive representation of sedentary behaviors and social support. It seems that sedentary behaviors of elderly can be determined by automatic and intentional processes. The older adults may also use cognitive strategies with the intent to compensate for sitting time through active behaviors and social support of older adults can contribute to the promotion of sedentary time or the interruption of the same. In this way, the understanding of psychosocial factors constitutes an important contribution to the explanation of the sedentary time of older adults.

Keywords: sedentary lifstyle, aging, epidemiologic factors.

\section{INTRODUÇÃO}

Nos últimos anos temos assistido a um crescimento progressivo acerca da investigação do comportamento sedentário (Chastin, Fitzpatrick, Andrews e DiCroce, 2014; Chastin et al., 2015; Van Dyck, Mertens, Cardon, De Cocker, \& Bourdeaudhuij, 2017; Palmer et al., 2018). Entende-se que o comportamento sedentário é caraterizado por um gasto energético inferior a 1,5 METs (equivalentes metabólicos) quando se está na posição sentada ou reclinada (Sedentary Behaviour Research Network, 2012). Isto significa que, por exemplo, quando um indivíduo está sentado na visualização de TV encontra-se em comportamento sedentário. No entanto, o termo comportamento sedentário não deve ser confundido com o baixo nível de prática de atividade física dos indivíduos; os indivíduos que não cumprem as recomendações de prática de atividade física de intensidade moderada a vigorosa são considerados inativos fisicamente e não indivíduos sedentários (Sedentary Behaviour Research Network, 2012). De facto, a investigação epidemiológica indica que as consequências para a saúde do excesso de tempo de comportamento sedentário são independentes da inatividade física, sendo um problema sério de saúde pública (Owen et al., 2011).

No que respeita à população idosa, as evidências indicam que o comportamento sedentário representam grande parte do seu dia-dia, estando em média 9.4 horas nesses comportamentos (Harvey, Chastin, \& Skelton, 2015). As evidências de literatura são claras quanto à relação positiva entre o excesso de tempo sentado e os riscos para a saúde biopsicossocial da população idosa. Nesse sentido, os resultados indicam associações positivas entre o demasiado tempo sentado e as doenças cardiovasculares (Stamatakis, Davis, Stathi, \& Hamer, 2012), o isolamento social 


\section{Determinantes psicossociais do comportamento sedentário dos idosos: estudo qualitativo}

(De Rezende, Rey-López, Matsudo, \& Do Carmo Luiz, 2014), o envelhecimento menos bem-sucedido (Dogra \& Stathokostas, 2012), a sintomatologia depressiva (Gardner, Lliffe, Fox, Jefferis, \& Hamer, 2014) e a morte prematura (Katzmarzyk, Church, Craig, \& Bouchard, 2009). Os dados mostram que a redução do tempo total sentado pode ser tão importante quanto o aumento da participação dos níveis de atividade física na diminuição de riscos da saúde (Katzmarzyk et al., 2009).

Nas últimas duas décadas verificou-se um aumento exponencial acerca do interesse teórico e prático dos modelos ecológicos. Este interesse surgiu devido à sua aplicação poder explicar a mudança dos diferentes comportamentos de saúde dos indivíduos (Sallis, Owen, \& Fisher, 2015). De acordo com Owen et al. (2011) o modelo ecológico assume uma grande importância para explicar as determinantes do comportamento sedentário. Sallis et al. (2015) destacam que uma abordagem ecológica para a compreensão das influências sobre um comportamento pressupõe vários níveis de influência, incluindo o indivíduo, o social, o organizacional/comunidade, o ambiental e o político. De particular importância é entender a configuração do comportamento, ou seja, o contexto físico e social onde ocorre (Barker, 1968). De facto, o comportamento sedentário pode ocorrer numa variedade de contextos (e.g., trabalho, lazer) e através de diversas atividades (ver TV, utilizar o computador, ler). Segundo esta perspetiva, o demasiado tempo sentado pode ser determinado pelos constrangimentos ambientais, por exemplo, dos escritórios, salas de aula, etc. Na tentativa de compreender os determinantes do comportamento sedentário ao longo da vida foi desenvolvido um consenso internacional que incluiu as prioridades de investigação nesse domínio (Chastin et al., 2016). Foram identificadas as seguintes linhas pesquisa acerca dos fatores determinantes do comportamento sedentário: 1) saúde física, 2) contexto social e cultural, 3) ambiente construído, 4) psicologia e comportamento, 5) política e economia e 6) ajustamentos nas configurações das habitações dos indivíduos. Identificar os determinantes do comportamento sedentário, especificamente aqueles que são modificáveis, como por exemplo os fatores psicológicos, é um passo necessário para o desenvolvimento de intervenções que permitam reduzir o comportamento sedentário do idoso (Chastin et al., 2015) e aumentar os seus níveis habituais de atividade física.

No entanto, verifica-se a existência de incrementar conhecimento empírico no que respeita a estudos de investigação qualitativa acerca dos fatores determinantes do comportamento sedentário do idoso. Os estudos anteriores sugerem que o comportamento sedentário do idoso pode ser determinado pela dor física, problemas de saúde e pela falta de energia para a realização de atividades físicas (Chastin et al., 2014; Dogra, Tam-Seto, \& Weir, 2016; GreenwoodHickman, Renz, \& Rosenberg, 2015). Os idosos também se sentam para gerir a sua condição de saúde e o gasto energético ao longo do dia (Chastin et al., 2014; Leask, Sandlund, Skelton, Tulle, \& Chastin, 2016). Outros determinantes do comportamento sedentário encontram-se relacionados com a pressão 


\section{Ramalho, A., Petrica, J., Rosado, A.}

familiar, dos amigos e dos cuidadores para que o idoso esteja sentado (Chastin et al., 2014, GreenwoodHickman et al., 2015; Mcewan, Tam-Seto, \& Dogra, 2016). Os idosos também consideram que a as condições atmosféricas e a falta de espaços verdes os limitam na realização de atividades físicas (Chastin et al., 2014; Greenwood-Hickman et al., 2015) e, também, a falta de informação sobre os malefícios do comportamento sedentário os levam a estar mais tempo sentados (Chastin et al., 2014; Dogra et al., 2016). As evidências também mostram que os idosos associam o comportamento sedentário a benefícios cognitivos/sociais, incorporando esses comportamentos nas suas rotinas diárias e, portanto, difíceis de mudar (Palmer et al., 2018).

Apesar dos estudos anteriores evidenciarem importantes fatores determinantes do comportamento sedentário do idoso, verifica-se a escassez na identificação de fatores psicológicos que também possam determinar o comportamento sedentário desta população. Noutro sentido, uma das lacunas do modelo ecológico consiste em não destacar o papel que as variáveis psicológicas podem desempenhar na explicação do comportamento sedentário, em detrimento do papel de destaque que é dado às variáveis contextuais. Desta forma, trabalhos de revisão de literatura destacam a necessidade de se investigar de que forma é que os fatores psicossociais determinam o comportamento sedentário do idoso (Chastin et al., 2015). Assim, é necessário construir um corpo de evidências mais robusto acerca dos fatores determinantes do comportamento sedentário (Owen, 2012) e os estudos futuros devem ser realizado no contexto da investigação qualitativa (Chastin et al., 2015). Deste modo, a pertinência deste estudo é composta por uma dimensão prática e teórica. No que respeita à prática, surgiu a necessidade de se investigar os fatores psicossociais determinantes do comportamento sedentário que possam permitir o desenvolvimento de intervenções mais eficazes na redução do tempo sentado dos idosos (Owen et al., 2011). Do ponto de vista teórico, os modelos ecológicos podem incorporar constructos psicossociais provenientes de outras investigações, com a intenção de desenvolver uma estrutura mais abrangente que permita a integração de múltiplas teorias tendo em consideração o contexto ambiental (Sallis et al., 2015). Desta forma, o objetivo do estudo foi identificar e compreender os fatores psicossociais que determinavam os diferentes comportamentos sedentários dos idosos.

\section{MÉTODO}

Este estudo foi abordado a partir de uma perspetiva interpretativa, cujo objetivo é representar a realidade através da interação entre os participantes e os investigadores de uma forma confiável (Guba \& Lincoln, 2005). Desta forma, assumiu-se uma ontologia relativista e uma epistemologia subjetivista.

\section{Participantes}

Os participantes foram recrutados pessoalmente, selecionados intencionalmente e por conveniência. Pretendeu-se a inclusão de indivíduos que eram adequados para responder ao problema de investigação (Marshall, 1996). Desta forma, na seleção dos participantes teve-se em conta os 


\section{Determinantes psicossociais do comportamento sedentário dos idosos: estudo qualitativo}

seguintes critérios de exclusão: luto nos últimos dois meses, doença terminal, sofrer de demência leve a avançada e apresentar alterações motoras e sensoriais que limitem a realização de atividades físicas diárias (Withall et al., 2014). Os critérios de seleção foram baseados na classificação da idade da pessoa idosa, sendo a partir dos 60 anos (WHO, 2016). No entanto, à medida que os dados começaram a ser analisados os participantes foram selecionados através da amostragem teórica, permitindo identificar padrões gerais e variações nos dados (Corbin \& Strauss, 2008). Assim, numa fase inicial foram recrutados idosos sem formação académica superior e não institucionalizados. Posteriormente, foram recrutados idosos com formação académica superior e igualmente não institucionalizados. Assim, foram recrutados idosos com diferentes características relacionadas com o género, idade e experiências profissionais.

Os participantes foram constituídos por vinte idosos (8 homens e 12 mulheres) com idades compreendidas entre os 65 e os 70 anos $(\mathrm{M}=68$ anos; $\mathrm{DP}=1.6)$. Os idosos residiam nas suas habitações $(\mathrm{N}=20)$ e oito dos participantes possuíam formação académica superior. Através do questionário de Cauwenberg, Holle, Bourdeudhuij, Owen e Deforche (2014) foi identificada a média do tempo de comportamento sedentário dos participantes ao longo do dia (7.9 horas). Foram consideradas as questões éticas, pelo que os participantes assinaram o consentimento informado.

\section{Recolha de dados}

Foram realizadas entrevistas individuais através de um guião de entrevista semiestruturada. O guião da entrevista foi submetido à avaliação do seu conteúdo através de um painel de especialistas em investigação qualitativa. A concordância acerca do conteúdo do guião da entrevista foi estabelecida através do acordo de consenso, entendida como uma estratégia que se utiliza em estudos observacionais no qual os observadores chegam a um acordo do que observar antes de se realizar a observação (Anguera, 1990; Morillo \& Hernández-Mendo, 2015). Posteriormente, o guião foi submetido a um estudo piloto com dois idosos não pertencentes ao grupo de participantes do estudo e revelou-se adequado. O guião da entrevista foi composto por questões gerais acerca dos fatores determinantes do comportamento sedentário (por exemplo., "Na sua opinião, quais os motivos que, ao longo do dia, o levam a estar sentado?”). No entanto, o guião da entrevista não representou um documento rígido, mas sim um conjunto evolutivo de questões (Corbin \& Strauss, 2008). Assim, existiram questões que foram desenvolvidas a partir dos dados emergentes, isto é, as respostas dos idosos influenciaram as questões subsequentes. A abordagem semiestruturada permitiu que a entrevista decorresse ao longo de uma conversa informal, mas dentro de um protocolo de entrevista. As entrevistas ocorreram nas habitações de cada idoso e foram registadas através de um gravador de áudio. No momento da realização das entrevistas estava presente o entrevistado e o primeiro autor. Foi realizada uma entrevista com cada participante com uma duração média de 38 minutos. $\mathrm{O}$ primeiro autor registou notas de campo acerca daquilo que refletiu durante as entrevistas.

Análise de dados 


\section{Ramalho, A., Petrica, J., Rosado, A.}

O conteúdo das entrevistas foi transcrito na íntegra para formato digital e foi analisado através de uma análise temática indutiva (Braun \& Clarke, 2006). O objetivo principal da análise temática consiste em identificar padrões num conjunto de dados, no sentido de os descrever e interpretar os seus significados (Braun, Clarke, \& Weate, 2016). A análise temática incluiu a familiarização com os dados, a definição de códigos iniciais, a classificação dos códigos em temas e a revisão e refinamento dos temas (Braun et al., 2016). A identificação de temas-chave não dependeu de medidas quantificáveis, mas sim de captar algo importante relativamente ao problema de investigação (Braun \& Clarke, 2006). Embora as perceções dos idosos fossem únicas, foram encontrados padrões semelhantes no conjunto da totalidade dos dados. Apesar de os dados terem sido analisados de forma indutiva, as interpretações também foram guiadas pelos conhecimentos teóricos dos autores acerca de diferentes constructos psicossociais. De facto, os investigadores não codificam os dados num vácuo epistemológico (Braun \& Clarke, 2006). Estes procedimentos de análise de dados estão de acordo com a premissa que a maioria das análises temáticas incluem elementos indutivos e dedutivos (Braun et al., 2016). A análise dos dados também seguiu alguns dos princípios das técnicas de análise de dados de estudos observacionais em psicologia do desporto (Anguera \& Hernández-Mendo, 2015; Argilaga, Villasñor, Mendo, \& López, 2011; Pérez-López, MoralesSánchez, Anguera, \& Hernández-Mendo, 2015). Desta forma, o objetivo da análise de dados foi contruir, a partir dos dados recolhidos, um sistema organizacional de categorias e de subcategorias que permitisse representar as perceções dos idosos entrevistados acerca dos determinantes do comportamento sedentário. Nesse sentido, o sistema de categorias desenvolvido apresentou diferentes categorias centrais e cada uma delas foi composta por diferentes subcategorias, correspondentes a manifestações relacionadas de cada categoria (Anguera \& Hernández-Mendo, 2013).

\section{Credibilidade dos dados}

Os dados das entrevistas foram triangulados com as notas de campo do primeiro autor. Outra técnica utilizada foi o peer debriefing (Lincoln \& Guba, 1985). Desta forma, durante o curso de investigação foram realizadas reuniões periódicas com a equipa de investigação para analisar criticamente todo o processo. Também se recorreu ao member checking (Lincoln \& Guba, 1985). Desta forma, os participantes receberam uma cópia escrita com as transcrições das suas entrevistas com a finalidade de as confirmar ou sugerir alterações. Depois, com a finalidade de avaliar a adequação dos temas emergentes dos dados, o primeiro autor reuniu-se com cada um dos idosos entrevistados. As observações dos idosos foram tomadas em consideração na interpretação dos dados. Além disso, foi realizada uma verificação da qualidade dos dados através de dois investigadores. Nesse sentido, numa primeira fase o primeiro autor codificou as transcrições das entrevistas até estar definido o sistema de categorias. Posteriormente, para cada uma das categorias, foi calculado o acordo de inter-juízes através do coeficiente de Kappa de Cohen. Desta forma, as categorias e as subcategorias do sistema foram categorizadas de forma a assegurar a sua 


\section{Determinantes psicossociais do comportamento sedentário dos idosos: estudo qualitativo}

exclusividade e exaustividade (Anguera \& Hernández-Mendo, 2013).

\section{RESULTADOS}

Os dados descritos na seção de resultados resultaram na identificação de diferentes categorias e de subcategorias (Tabela 1). O sistema de categorias apresenta a frequência com que cada categoria e subcategoria foram referenciadas no decorrer das entrevistas realizadas. As categorias emergentes dos dados foram apresentados através de uma narrativa analítica, num nível de análise semântico, e as citações Tabela 1

Sistema de categorias e de subcategorias dos determinantes psicossociais do comportamento sedentário dos idosos

\begin{tabular}{|c|c|c|}
\hline $\begin{array}{c}\text { Definição do } \\
\text { núcleo categorial }\end{array}$ & Subcategorias & Comportamentos Sedentários \\
\hline $\begin{array}{l}\text { 1.Hábito }(\mathrm{n}=81) \text { : o } \\
\text { comportamento } \\
\text { sedentário do } \\
\text { idoso pode ser } \\
\text { influenciado } \\
\text { através dos hábitos } \\
\text { sedentários } \\
\text { desenvolvidos ao } \\
\text { longo dos anos }\end{array}$ & $\begin{array}{l}\text { 1.1.Hábito do comportamento sedentário regulado pela } \\
\text { hora do dia }(n=42) \\
\text { 1.2.Hábito do comportamento sedentário regulado pelo } \\
\text { contexto da residência }(n=39)\end{array}$ & $\begin{array}{l}\text { Visualização de TV; ouvir } \\
\text { música; utilização do computador; } \\
\text { atividades cognitivas (por } \\
\text { exemplo, palavras cruzadas, ler) }\end{array}$ \\
\hline $\begin{array}{l}\text { 2.Comportamentos } \\
\text { compensatórios } \\
(\mathrm{n}=77) \text { : estratégias } \\
\text { cognitivas que o } \\
\text { idoso utiliza para } \\
\text { não se sentir } \\
\text { culpado do } \\
\text { excesso de tempo } \\
\text { que está sentado }\end{array}$ & $\begin{array}{l}\text { 2.1.Tempo sentado é compensado pela vida diária ativa } \\
\text { (por exemplo, ir às compras) }(n=24) \\
\text { 2.2.Tempo sentado é compensado pela prática regular de } \\
\text { atividade física }(n=34) \\
\text { 2.3.Tempo sentado é compensado pelas interrupções do } \\
\text { comportamento sedentário }(n=19)\end{array}$ & $\begin{array}{l}\text { Visualização de TV; sentado em } \\
\text { repouso; sentado em conversa } \\
\text { social; atividades manuais (por } \\
\text { exemplo, bricolage; costurar) }\end{array}$ \\
\hline $\begin{array}{l}\text { 3. Representação } \\
\text { positiva dos } \\
\text { comportamentos } \\
\text { sedentários } \\
\text { (n=75): avaliação } \\
\text { positiva que o } \\
\text { idoso faz daqueles } \\
\text { comportamentos } \\
\text { sedentários que } \\
\text { considera que são } \\
\text { benéficos }\end{array}$ & $\begin{array}{l}\text { 3.1.Comportamento sedentário proporciona bem-estar } \\
(\mathrm{n}=43) \\
\text { 3.2.Comportamento sedentário proporciona a ocupação do } \\
\text { tempo livre }(\mathrm{n}=32)\end{array}$ & $\begin{array}{l}\text { Visualização de TV; utilização do } \\
\text { computador; atividades cognitivas } \\
\text { (por exemplo, palavras cruzadas, } \\
\text { ler); atividades manuais (por } \\
\text { exemplo, bricolage; costurar) }\end{array}$ \\
\hline
\end{tabular}

dos participantes foram apresentadas no texto quando apropriado. Os resultados dos índices de concordância inter-juízes, para cada categoria, calculados através do coeficiente de Kappa de Cohen foram os seguintes: 1) Hábito (0.85); 2) Comportamentos compensatórios $(0.80)$; 3$)$ Representação positiva dos comportamentos sedentários (0.81); 4) Suporte social (0.83). Fleiss (1981) carateriza os valores de kappa como pobres se $<0.40$, de satisfatório a bom quando os valores se situam entre o 0.40-0.75, e valores excelentes quando $>$ 0.75 . 


\section{Ramalho, A., Petrica, J., Rosado, A.}

\begin{tabular}{|c|c|c|}
\hline $\begin{array}{l}\text { 4. Suporte social } \\
\text { (n=70): refere-se à } \\
\text { influência do } \\
\text { contexto familiar e } \\
\text { do círculo de } \\
\text { amigos que podem } \\
\text { determinar o } \\
\text { comportamento } \\
\text { sedentário do } \\
\text { idoso }\end{array}$ & $\begin{array}{l}\text { 4.1.Família estimula o comportamento sedentário }(\mathrm{n}=28) \\
\text { 4.2.Família desencoraja o comportamento sedentário } \\
(\mathrm{n}=15) \\
\text { 4.3.Amigos estimulam o comportamento sedentário } \\
(\mathrm{n}=21) \\
\text { 4.4.Amigos desencorajam o comportamento sedentário } \\
(\mathrm{n}=6)\end{array}$ & $\begin{array}{l}\text { Visualização de TV; sentado em } \\
\text { repouso; sentado em conversa } \\
\text { social; atividades manuais (por } \\
\text { exemplo, costurar) }\end{array}$ \\
\hline
\end{tabular}

\section{Hábito}

Os participantes $(\mathrm{n}=16)$ identificaram que o seu comportamento sedentário era determinado pelos seus hábitos e rotinas diárias, de acordo com a hora do dia. Nesse sentido, eles consideraram que "Tenho sempre o hábito de me sentar para ver TV seguir ao almoço. Há muitos anos que é assim (...). Sou capaz de lá estar quase duas horas. " (Participante 8). Com a mesma perspetiva de que estar sentado era um hábito diário, outros participantes observaram que depois do jantar a rotina era ver TV porque "Há muito tempo que eu e o meu marido vemos as novelas juntos. É quase como se fosse uma tradição." (Participante 10). Outros idosos $(\mathrm{n}=9)$ consideraram que quando se sentiam melancólicos o seu hábito era sentar-se para poderem refletirem acerca das suas preocupações: "Sempre que me sinto triste, sento-me para tentar por as ideias no sítio." (Participante 14). Noutro sentido, outros participantes $(\mathrm{n}=14)$ percecionaram que tinham o hábito de se sentar sempre que estavam em algumas zonas das suas habitações: "Se eu estiver na sala é quase certo que me vou sentar logo. Estou habituado a isso. A TV está lá e a tendência é para a pessoa se sentar". (Participante 18).

Comportamentos compensatórios
Doze dos participantes consideraram que o demasiado tempo sedentário era prejudicial para a sua saúde física: "Sei que estar sentado não é muito bom para a saúde das pessoas. Faz-me dores no corpo quando estou muito tempo sentado." (Participante 16). Contundo, eles tinham a perceção que os riscos para a saúde do demasiado tempo sentados eram compensados pela vida ativa que mantinham. Desta forma, os idosos entendiam que dar suporte familiar (e.g., cuidar dos netos), participar em atividades sociais (e.g., ações de solidariedade), entre outras atividades, era mais do que suficientes para compensar os efeitos negativos do excesso de tempo sentados: "Até posso estar a maior parte do dia sentado, mas como me vou ocupando ao longo do dia e vou caminhando, não há grande problema em estar muito tempo sentado. Até é bom para descansar." (Participante 4). No mesmo sentido, outros participantes $(n=10)$ identificaram que os efeitos negativos do seu comportamento sedentário eram equilibrados através da prática regular de atividade física: "Eu sei que não é saudável estar muito tempo sentado. Mas como, durante a semana, faço aulas de hidroginástica e de atividade fisica, acho que isso permite-me que possa estar sentado durante algum tempo." (Participante 12). Outra citação que reforça a 


\section{Determinantes psicossociais do comportamento sedentário dos idosos: estudo qualitativo}

mesma ideia foi proferida por outro idoso: "Como quase todos os dias pratico exercício físico e faço caminhadas, se ficar sentado por umas horas não tem grande mal. Uma coisa equilibra a outra." (Participante 6). Os participantes $(\mathrm{n}=16)$ também reconheceram que os malefícios do tempo sentado podiam ser equilibrados através de interrupções: "Estou sentada por umas horas, depois levanto-me um pouco para ir esticar as pernas. Depois volto a sentarme durante algum tempo e fico bem assim. Pode fazer mal, mas assim acho que não faz tanto." (Participante 4). Os dados também indicaram que a compensação dos comportamentos sedentários através de comportamentos ativos podia influenciar $\mathrm{o}$ desenvolvimento do hábito de estar sentado: "Como tenho o hábito de ler sempre um bocadinho depois do almoço, é claro que vou sempre arranjar forma que isso aconteça (...). Depois tenho tempo para caminhar um bocado, que também faz bem." (Participante 15).

Representação positiva dos comportamentos sedentários

Todos os entrevistados $(n=20)$ consideraram que o seu comportamento sedentário era determinado pelo bem-estar que estes proporcionavam. Nesse sentido, os idosos identificaram que "Gosto de ver TV. É um hábito que me faz bem à mente e por isso é indispensável no meu dia-a-dia." (Participante 15). Outros idosos tinham a leitura como um dos hobbies favoritos e por essa razão acumulavam períodos de tempo contínuo sentados ao longo do dia: "Para mim ler é fundamental. Não consigo passar um dia sem ler e para isso tenho que estar sentada não é?" (Participante 8). Na mesma linha de pensamento, outros participantes identificaram que outras atividades das suas preferências (e.g., bricolage, escrever, palavras cruzadas, tricot, ouvir música, etc.) determinavam o seu tempo de comportamento sedentário. Os idosos também transmitiram que quanto mais gostavam da atividade sedentária, mais tempo e mais frequentemente as realizavam ao longo do dia. Os idosos também transmitiram que quando realizavam atividades sedentárias do seu agrado, tinham a perceção que o tempo passava mais rápido do que o normal: "Às vezes nem dou conta que estou sentada tanto tempo seguido. Estou tão entretida na leitura, que se passam horas a fio enquanto estou ali." (Participante 4).

\section{Suporte social}

Oito idosos consideraram que o comportamento sedentário era determinado pelo seu ambiente familiar. Nesse sentido, as famílias dos idosos, na sua maioria os filhos, estimulavam que os idosos estivessem sentados com receio das quedas: "Os meus dois filhos ligam-me muitas vezes durante o dia (...). Pedem-me sempre para não me mexer muito, porque já cai uma vez e foi muito grave. (...) Sigo aquilo que eles dizem claro." (Participante 5). Com a mesma perspetiva, outros familiares expressavam as suas preocupações com o nível de saúde física dos idosos e por isso incitavam que estes pudessem estar sentados: "Sabe, há uns anos atrás ganhei uma dor nas costas e a minha filha está sempre a dizer-me para não me esforçar demasiado cá em casa e repousar. (...) Vou fazendo mas depois lembro-me do que ela me diz e fico mesmo em repouso." (Participante 6). No entanto, outros participantes $(\mathrm{n}=12)$ expressaram que as suas 


\section{Ramalho, A., Petrica, J., Rosado, A.}

famílias incentivavam que o tempo de comportamento sedentário fosse reduzido através da prática de atividade física: "O meu neto e o meu filho estão sempre a dizer-me para ir caminhar, que estar sempre sentada faz-me mal ao corpo e posso ter um AVC (acidente vascular cerebral). Às vezes até vou caminhar com eles e gosto muito." (Participante 18). No mesmo sentido, os idosos também consideraram que tinham amigos que os estimulavam a praticar mais exercício físico e a realizarem mais tarefas diárias, evitando estar muito tempo sentados.

\section{DISCUSSÃO}

O objetivo do estudo foi identificar os fatores psicossociais que determinavam os diferentes comportamentos sedentários dos idosos. Os resultados indicaram os seguintes fatores psicossociais determinantes: hábito, comportamentos compensatórios, representação positiva dos comportamentos sedentários e o suporte social. Nesta fase, procedeu-se à interpretação dos resultados no sentido de os teorizar em relação à literatura anterior (Braun \& Clarke, 2006).

Os resultados deste estudo parecem estar de acordo que os idosos incorporam os diferentes comportamentos nas suas rotinas diárias (Palmer et al., 2018). Nesse sentido, os hábitos podem ser adquiridos através de um emparelhamento repetido entre uma sugestão contextual e uma resposta comportamental, de forma que, ao longo do tempo, quando surgir uma determinada sugestão contextual é gerada, automaticamente, uma resposta comportamental (Aarts, Paulussen, \& Schaalma, 1997). De facto, os resultados deste estudo sugeriram que o comportamento sedentário dos idosos podia ser determinados através de processos automáticos como o hábito, em função de sugestões contextuais como a hora do dia ou do contexto da residência. Pesquisas emergentes mostram que os hábitos são um mecanismo importante pelo qual as pessoas autorregulam o seu comportamento (Wood, 2017). Desta forma, os resultados deste estudo parecem estar de acordo que os indivíduos podem desenvolver associações entre as atividades diárias e estarem sentados (por exemplo, estar sentado quando se vê TV) (Neal, Wood, \& Quinn, 2006). Assim, é sugerido que os idosos deste estudo mostraram que tinham desenvolvido hábitos de comportamento sedentário, uma vez que tiveram tempo para desenvolver associações entre sugestões contextuais (por exemplo, hora do dia e contexto da residência) e o comportamento sedentário (Verplanken, Walker, Davis, \& Jurasek, 2008). Outras evidências concluíram a existência de associações positivas entre o hábito e o comportamento sedentário de idosos, sugerindo que os comportamentos sedentários são regulados através de processos automáticos e intencionais (Maher \& Conroy, 2016). Nesse sentido, as evidências empíricas sugerem que as respostas comportamentais automáticas em função de sugestões contextuais podem ser conscientemente inibidas (Quinn, Pascoe, Wood, \& Neal, 2010). Assim, os hábitos também podem ser controlados através de processos de autorregulação (Neal, Wood, \& Drolet, 2013), sugerindo que o comportamento sedentário dos 


\section{Determinantes psicossociais do comportamento sedentário dos idosos: estudo qualitativo}

idosos não era exclusivamente realizado através de processos automáticos, mas também através de processos intencionais (por exemplo, sento-me porque gosto). Nesse sentido, parece que a autodeterminação pode desempenhar um papel importante na automatização dos comportamentos realizados frequentemente (Radel, Pelletier, Pjevac, \& Cheval, 2017). Desta forma, os hábitos do comportamento sedentário dos idosos podem ser vistos como um processo pelo qual um estímulo gera automaticamente um impulso para a ação, com base em associações de estímulo-resposta aprendidas.

As evidências deste estudo indicaram que os idosos acreditavam que o comportamento sedentário era prejudicial para a saúde, mas, ao longo do dia, estavam muito tempo sentados. Nesse sentido, sugere-se que os comportamentos dos idosos não estavam de acordo com as suas crenças. No entanto, os indivíduos tendem a procurar uma coerência entre as suas crenças e os seus comportamentos (Cooper, 2007). Porém, quando as crenças do individuo entram em conflito com as suas ações comportamentais estamos perante a uma dissonância cognitiva (Cooper, 2007). No caso deste estudo, sugere-se que os idosos utilizavam estratégias cognitivas para reduzir a dissonância cognitiva, justificando as escolhas de comportamentos não saudáveis (por exemplo, comportamentos sedentários) com a intenção de se envolverem em comportamentos saudáveis (por exemplo, praticar atividade física, interrupções do comportamento sedentário). De facto, resultados anteriores mostraram que os idosos podiam diminuir o seu nível de atividade física através das perceções de compensação dos seus comportamentos ativos (Gray, Murphy, Gallagher, \& Simpson, 2017). Os resultados do presente estudo também parecem estar de acordo com os princípios teóricos do Compensatory Health Beliefs (CHBs) (Rabiau, Knäuper, \& Miquelon, 2006). Este modelo teórico propõe que os indivíduos acreditam que os efeitos negativos de um comportamento não saudável e prazeroso podem ser compensados através de um comportamento saudável (Rabiau et al., 2006) (por exemplo, posso estar muito tempo sentado porque depois vou praticar exercício). Nesse sentido, a tendência dos indivíduos é a procura de um equilíbrio entre a satisfação dos seus desejos e o cumprimento dos seus objetivos, mesmo que estes sejam contraditórios em relação aos seus desejos (Muraven \& Baumeister, 2000). Desta forma, quando a satisfação dos desejos dos idosos (por exemplo, estar sentado) está em desacordo com os seus objetivos (por exemplo, serem fisicamente mais ativos), poderá existir um sentimento de culpa antecipada. Esse sentimento de culpa poderá despoletar que os idosos procurem estratégias cognitivas que o permita aliviar. Nesse sentido, as ativações das crenças de saúde compensatórias podem ser eficazes na atenuação dos sentimentos de culpa dos indivíduos (Rabiau et al., 2006). Noutro sentido, os resultados também mostraram que os idosos apreciavam a realização de diversas atividades sedentárias. Estes resultados parecem estar em concordância de que a utilização das estratégias cognitivas, como as crenças de saúde compensatórias, podem ser ativadas quando o resultado do comportamento desejado é satisfatório para os indivíduos (Norman, Conner, \& Bell, 1999). 


\section{Ramalho, A., Petrica, J., Rosado, A.}

Os resultados sugeriram que os idosos manifestaram uma representação positiva acerca do tempo utilizado em diferentes comportamentos sedentários. Entendemos que os idosos encaravam a realização de alguns comportamentos sedentários como parte essencial na sua rotina diária. No entanto, é preciso ter em consideração que muitos dos comportamentos sedentários eram de fácil realização para os idosos entrevistados, possibilitando que estes obtivessem sucesso na atividade sedentária que realizavam. Nesse sentido, oportunidades de ação dos diversos comportamentos sedentários estavam em equilíbrio com as habilidades dos idosos. De facto, a realização de diversos comportamentos sedentários, como a visualização de TV, atividades de leitura e ouvir música estão associadas ao divertimento dos idosos (Heo, Lee, Pedersen, \& McCormick, 2010). Desta forma, os comportamentos sedentários contemplam componentes cognitivas e sociais que são benéficas para o bem-estar subjetivo, proporcionando a satisfação de necessidades psicológicas, sociais e de relaxamento (Östlund, 2010). Portanto, o comportamento sedentário dos idosos pode ser determinado por valores, interesses e objetivos e pelos níveis de prazer que proporcionam (Salmon, Owen, Crawford, Bauman, \& Sallis, 2003). Nesse sentido, entendemos que quando os idosos percecionam que determinados comportamentos sedentários são benéficos, é natural que os realizem sistematicamente por longos períodos de tempo. Desta forma, poderá ser possível que os níveis de prazer e de divertimento que os entrevistados evidenciaram possam ser associados a um protótipo de um comportamento autodeterminando. De facto, existem evidências que mostram que os constructos motivacionais subjacentes ao lazer/recreação encontram-se associados ao comportamento sedentário dos indivíduos (Gaston, De Jesus, Markland, \& Prapavessis, 2016).

Relativamente ao suporte social dos idosos (família e amigos) entendemos que podia atuar em dois sentidos: desencorajar o comportamento sedentário e promover o comportamento sedentário. Desta forma, sugere-se que as opiniões fornecidas a partir da interação com as pessoas mais próximas podem influenciar $o$ comportamento dos idosos, parecendo ser possível que os idosos interrompessem o comportamento sedentário por influência dos seus familiares. No que respeita à promoção do comportamento sedentário, sugere-se que as preocupações dos familiares com a saúde dos idosos podiam funcionar como uma variável determinante do tempo sentado. Os resultados deste estudo parecem estar de acordo com as evidências anteriores que mostraram que pode existir pressão por parte dos familiares, dos amigos e dos cuidadores para que os idosos se sentem ao longo do dia (Chastin et al., 2014, Greenwood-Hickman et al., 2015; McEwan et al., 2016). No mesmo sentido, outras evidências concluíram que o suporte social pode possibilitar uma diminuição da prática de atividade física dos idosos (Van Dyck, Cardon, \& De Bourdeaudhuij, 2017).

Umas das limitações deste estudo foi a realização de entrevistas individuais ao invés da utilização de grupos focais. Nesse sentido, investigações futuras podem incluir métodos que permitam múltiplas interações com os participantes, uma vez que são necessárias mais investigações qualitativas acerca dos determinantes do comportamento sedentário dos 


\section{Determinantes psicossociais do comportamento sedentário dos idosos: estudo qualitativo}

idosos (Chastin et al., 2015). O facto deste estudo ter recolhido dados através de uma única entrevista constitui-se noutra limitação. Desta forma, é recomendável que os estudos futuros interpretativos adotem a realização de entrevistas longitudinais, por permitir analisar a natureza dinâmica dos pensamentos dos participantes ao longo do tempo. Serão também necessários estudos de natureza quantitativa que permitam explorar os resultados desta investigação. Nesse sentido, os estudos futuros acerca dos determinantes psicossociais do tempo sedentário podem ser realizados no contexto das evidências observacionais e experimentais. Desta forma, os estudos futuros podem passar pela avaliação da satisfação e da frustração das necessidades psicológicas básicas dos idosos, bem como a forma como estes regulam a sua motivação na realização de determinados comportamentos sedentários. Também seria pertinente investigar a relação entre as diferentes posições teóricas acerca do hábito e os comportamentos sedentários dos idosos, especificando o tipo de comportamento. Uma última recomendação consiste na verificação da utilidade do modelo teórico CHBs (Rabiau et al., 2006) na explicação do comportamento sedentário do idoso. Outra limitação diz respeito ao presente estudo ter apenas investigado as relações entre os determinantes psicossociais do comportamento sedentário dos idosos. Seria também importante que os estudos futuros se centrassem nas relações entre todos os grupos de determinantes do comportamento sedentário que o modelo ecológico de Owen et al. (2011) sugere.
Em conclusão, os comportamentos sedentários dos idosos podem ser determinados através de processos automáticos, como o hábito, $\mathrm{e}$ de processos intencionais (por exemplo, sento-me porque gosto). Nesse sentido, parece que determinados comportamentos sedentários possuem características que os idosos apreciam, contribuindo para a sua realização por longos períodos de tempo interruptos. Desta forma, parece que quando os idosos realizam comportamentos sedentários cujo grau de importância é significativo para eles, pode existir uma distorção temporal (perceção que o tempo passou mais rápido que o normal). Noutro sentido, os idosos também podem utilizar estratégias cognitivas que para que não se sintam culpados do excesso de tempo que estão sentados. Assim, parece que os idosos percecionam que o excesso de tempo sentado pode ser compensado através de comportamentos ativos, como por exemplo a prática de atividade física. No que respeita ao suporte social, parece que as preocupações da família com o estado de saúde dos idosos pode contribuir para a promoção do tempo sedentário. Em sentido contrário, o suporte familiar e os amigos também podem contribuir para que os idosos interrompam mais frequentemente o tempo sedentário e tenham uma vida fisicamente mais ativa. $\mathrm{O}$ entendimento dos fatores psicossociais constitui-se numa importante contribuição para a explicação do tempo sedentário dos idosos, à semelhança do que acontece para na compreensão da prática de exercício físico (Pardo, Castrillón, Pedreño, \& Moreno-Murcia, 2014).

\section{IMPLICAÇÕES PRÁTICAS}




\section{Ramalho, A., Petrica, J., Rosado, A.}

Este estudo permitiu entender, em parte, a complexidade que caracteriza os problemas de saúde pública, como o excessivo tempo sedentário que a população idosa revela. Como tal, os resultados deste estudo podem contribuir para o desenvolvimento de intervenções comportamentais que permitam uma redução do tempo sedentário dos idosos. Estes resultados poderão ser particularmente úteis para os profissionais de diferentes áreas de intervenção geriátrica (e.g., médicos, psicólogos, fisioterapeutas, técnicos superiores de atividade física). Os resultados das investigações anteriores mostraram que existe uma relação positiva entre o excesso de tempo em comportamento sedentário e os riscos para a saúde dos idosos. Nesse sentido, tomando em consideração que os idosos podem ter a perceção que o excesso de tempo de comportamento sedentário pode ser compensado através de comportamentos ativos, como a prática de atividade física durante 30 minutos por dia, poderá ser pertinente clarificar a população idosa sobre a importância para a saúde de interromperem os longos períodos de tempo ininterruptos de comportamento sedentário ao longo do dia (por exemplo, realizar atividades domésticas ao longo do dia). De facto, uma redução do volume total do comportamento sedentário pode ser relevante para que possa existir uma diminuição dos riscos de saúde (Katzmarzyk et al., 2009). Nesse sentido, as intervenções podem aproveitar as interrupções do comportamento sedentário que os idosos já realizam para promover mudanças comportamentais sustentáveis (Chastin et al., 2014).
No mesmo sentido, os idosos também devem ser instruídos a adquirem um equilíbrio entre o tempo de realização das atividades sedentárias que mais gostam e a interrupção das mesmas. No entanto, os idosos também devem ser estimulados a realizarem aquelas atividades sedentárias que podem ser estimulantes do ponto de vista psicossocial (por exemplo, atividades cognitivas como ler ou sudoku), prevenindo o envelhecimento cerebral (Kesse-Guyot et al., 2012). Assim, as intervenções não devem considerar interromper os períodos sedentários que os idosos considerem benéficos (Chastin et al., 2014). Uma outra estratégia que poderá permitir uma redução do tempo total do comportamento sedentário dos idosos poderá ser o estímulo para que estes desenvolvam novas conexões sociais (por exemplo, grupos comunitários que pratiquem regularmente exercício físico). Os familiares e os cuidadores dos idosos também poderão ser alvos de formação acerca dos malefícios para a saúde do demasiado tempo em comportamento sedentário para que não inibam os idosos da realização de atividade física ao longo do dia. No que respeita ao hábito dos idosos na realização de diversos comportamentos sedentários, este poderia ser controlado através de sugestões de ação de movimento via telemóvel. Esta estratégia resultou na mudança de hábitos de saúde dos indivíduos (Mullan, Allom, Fayn, \& Johnston, 2014).

\section{REFERÊNCIAS}

1. Aarts, H., Paulussen, T., \& Schaalma, H. (1997). Physical exercise habit: On the conceptualization and formation of habitual health behaviours. Health Education 


\section{Determinantes psicossociais do comportamento sedentário dos idosos: estudo qualitativo}

Research, $12, \quad 363-374 . \quad$ doi: $10.1093 /$ her/12.3.363

2. Anguera, M.T. (1990). Metodología Observacional. In J. Arnau, M.T. Anguera, \& J. Gómez (Eds.). Metodología de la Investigación en Ciencias del Comportamiento (pp. 125-236). Murcia: Secretariado de Publicaciones de la Universidad de Murcia.

3. Anguera, M.T., \& Hernández-Mendo, A. (2013). La metodología observacional en el ámbito del deporte. e-balonmano.com: Revista de Ciencias del Deporte, 9, 135-160.

4. Anguera, M.T., Hernández-Mendo, A. (2015). Técnicas de análisis en estudios observacionales en ciencias del deporte. Cuadernos de Psicología del Deporte,15, 1330.

5. Argilaga, M.T., Villaseñor, A., Mendo, A., \& López, J. (2011). Diseños observacionales: ajuste y aplicación en psicología del deporte. Cuadernos de Psicología del Deporte,11, 6376.

6. Barker, R. (1968). Ecological Psychology. Stanford, Calif.: Stanford University Press.

7. Braun, V., \& Clarke, V. (2006). Using thematic analysis in psychology. Qualitative Research in Psychology, 3, 77-101. doi: 10.1191/1478088706qp063oa

8. Braun, V., Clarke, V., \& Weate, P. (2016). Using thematic analysis in sport and exercise research. In B. Smith \& A. Sparkes (Eds.), Routledge Handbook of Qualitative Research in Sport and Exercise (pp. 191-205). Abingdon: Routledge.

9. Chastin, S., Buck, C., Freiberger, E., Murphy, M., Brug, J., Cardon, G., ... Oppert, J. (2015). Systematic literature review of determinants of sedentary behaviour in older adults: a DEDIPAC study. International Journal of Behavioral Nutrition and Physical Activity, 12:127. doi: 10.1186/s12966-015-0292-3

10. Chastin, S., De Craemer, M., Lien, N., Bernaards, C., Buck, C., Oppert, J., ... Cardon, G. (2016). The SOS-framework (Systems of Sedentary behaviours): an international transdisciplinary consensus framework for the study of determinants, research priorities and policy on sedentary behaviour across the life course: a DEDIPACstudy. International Journal of Behavioral Nutrition and Physical Activity, 13:83. doi: $10.1186 / \mathrm{s} 12966-016-0409-3$

11. Chastin, S., Fitzpatrick, N., Andrews, M., \& DiCroce, N. (2014). Determinants of Sedentary Behavior, Motivation, Barriers and Strategies to Reduce Sitting Time in Older Women: A Qualitative Investigation. International Journal of Environmental Research and Public Health, 11, 773-791. doi: $10.1186 / \mathrm{s} 12966-015-0292-3$

12. Cooper, J. (2007). Cognitive dissonance: 50 years of a classic theory. London: Sage.

13. Corbin, J., \& Strauss, A. (2008). Basics of qualitative research: Grounded theory procedures and techniques (3rd ed.). London: Sage. 


\section{Ramalho, A., Petrica, J., Rosado, A.}

14. De Rezende, L., Rey-López, J., Matsudo, V., \& Do Carmo Luiz, O. (2014). Sedentary behavior and health outcomes among older adults: a systematic review. BMC Public Health, 14:333. doi: 10.1186/1471-2458-14333

15. Dogra, S., \& Stathokostas, L. (2012). Sedentary behavior and physical activity are independent predictors of successful aging in middle-aged and older adults. Journal of Aging Research, 2012, 1-8. doi: $10.1155 / 2012 / 190654$

16. Dogra, S., Tam-Seto, L., \& Weir, P. (2016). Factors influencing sedentary behaviour in older adults: An ecological approach. AIMS Public Health, 3, 555-572. doi:10.3934/publichealth.2016.3.555

17. Fleiss, J. (1981). Statistical methods for rates and proportions (2th ed.). New York: John Wiley \& Sons.

18. Gardner, B., Lliffe, S., Fox, K., Jefferis, B., \& Hamer, M. (2014). Sociodemographic, behavioural and health factors associated with changes in older adults'TV viewing over 2 years. International Journal of Behavioral Nutrition and Physical Activity, 11:102. doi: 10.1186/s12966-014-0102-3

19. Gaston, A., De Jesus, S., Markland, D., \& Prapavessis, H. (2016). I sit because I have fun when I do so! Using self-determination theory to understand sedentary behavior motivation among university students and staff. Health
Psychology and Behavioral Medicine, 4, 138154. doi: 10.1080/21642850.2016.1170605

20. Gray, P., Murphy, M., Gallagher, A., \& Simpson, E. (2017). A qualitative investigation of physical activity compensation among older adults. British Journal of Health Psychology, 23, 208-224. doi: 10.1111/bjhp.12282.

21. Greenwood-Hickman, M., Renz, A., \& Rosenberg, D. (2015). Motivators and barriers to reducing sedentary behavior among overweight and obese older adults. The Gerontologist, $\quad 56, \quad 660-668$. doi:10.1093/geront/gnu163

22. Guba, E., \& Lincoln, Y. (2005). Paradigmatic controversies, contradictions, and emerging confluences. In N.K. Denzin \& Y.S. Lincoln (Eds.), The Sage handbook of qualitative research (pp. 191-215). Thousand Oaks, CA: Sage.

23. Harvey, J., Chastin, S., \& Skelton, D. (2015). How sedentary are older people? A systematic review of the amount of sedentary behavior. Journal of Aging and Physical Activity, 23, 471-487. doi: 10.1123/japa.2014-0164

24. Heo, J., Lee, Y., Pedersen, P., \& McCormick, B. (2010). Flow experience in the daily lives of older adults: an analysis of the interaction between flow, individual differences, serious leisure, location, and social context. Canadian Journal on Aging, 29, 411-423. doi:10.1017/S0714980810000395 


\section{Determinantes psicossociais do comportamento sedentário dos idosos: estudo qualitativo}

25. Katzmarzyk, P., Church, A., Craig, C., \& Bouchard, C. (2009). Sitting time and mortality from all causes, cardiovascular disease, and cancer. Medicine and Science in Sports and Exercise, 41, 998-1005. doi: 10.1249/MSS.0b013e3181930355

26. Kesse-Guyot, E., Charreire, H., Andreeva, V., Touvier, M., Hercberg, S., Galan, P., \& Oppert, J. (2012). Cross-Sectional and longitudinal associations of different sedentary behaviors with cognitive performance in older adults. PLoS ONE, 7: e47831. doi: 10.1371/journal.pone.0047831

27. Leask, C., Sandlund, M., Skelton, D., Tulle, E., \& Chastin, S. (2016). Modifying Older Adults' Daily Sedentary Behaviour Using an Asset-based Solution: Views from Older Adults. AIMS Public Health, 3, 542-554. doi: 10.3934/publichealth.2016.3.542

28. Lincoln, Y., \& Guba, E. (1985). Naturalistic inquiry. Newbury Park, CA: Sage.

29. Maher, J., \& Conroy, D. (2016). A dualprocess model of older adults' sedentary behavior. Health Psychology, 35, 262-272. doi: $10.1037 /$ hea0000300

30. Marshall, M. (1996). Sampling for qualitative research. Family Practice, 13, 522-526. doi: 10.1093/fampra/13.6.522

31. Mcewan, T., Tam-Seto, L., \& Dogra, S. (2016). Perceptions of Sedentary Behavior Among Socially Engaged Older Adults. The Gerontologist, $\quad 57, \quad 735-744 . \quad$ doi: 10.1093/geront/gnv689
32. Morillo, J., \& Hernández-Mendo, A. (2015). Análisis de la calidad del dato de un instrumento para la observación del ataque en balonmano playa. Revista Iberoamericana de Psicología del Ejercicio y el Deporte, 10, 1522.

33. Mullan, B., Allom, V., Fayn, K., \& Johnston, I. (2014). Building habit strength: A pilot intervention designed to improve food-safety behavior. Food Research International, 66, 274-278. doi: 10.1016/j.foodres.2014.09.027

34. Muraven, M., \& Baumeister, R. (2000). Selfregulation and depletion of limited resources: Does self-control resemble a muscle? Psychological Bulletin, 126, 247-259.

35. Neal, D., Wood, W., \& Drolet, A. (2013). How do people adhere to goals when willpower is low? The profits (and pitfalls) of strong habits. Journal of Personality and Social Psychology, 104, 959-975. doi: $10.1037 / \mathrm{a} 0032626$

36. Neal, D., Wood, W., \& Quinn, J. (2006). Habits-A repeat performance. Current Directions in Psychological Science, 15, 198202. doi: 10.1111/j.1467-8721.2006.00435.x

37. Norman, P., Conner, M., \& Bell, R. (1999). The theory of planned behaviour and smoking cessation. Health Psychology, 18, 89-94.

38. Östlund, B. (2010). Watching television in later life: A deeper understanding of $\mathrm{TV}$ viewing in the homes of old people and in geriatric care contexts. Scandinavian Journal 


\section{Ramalho, A., Petrica, J., Rosado, A.}

of Caring Sciences, 24, 233-243. doi: 10.1111/j.1471-6712.2009.00711.x

39. Owen, N. (2012). Sedentary behavior: Understanding and influencing adults' prolonged sitting time. Preventive Medicine, 55, 535-539. doi: 10.1016/j.ypmed.2012.08.024

40. Owen, N., Sugiyama, T., Eakin, E., Gardiner, P., Tremblay, M., \& Sallis, J. (2011). Adults' sedentary behavior determinants and interventions. American Journal of Preventive Medicine, 41, 189-196. doi: 10.1016/j.amepre.2011.05.013

41. Palmer, V., Gray, C., Fitzsimons, C., Mutrie, N., Wyke, S., Deary, I., ... Skelton, D. (2018). What Do Older People Do When Sitting and Why? Implications for Decreasing Sedentary Behavior. The Gerontologist, 0, 1-12. doi: 10.1093/geront/gny020

42. Pardo, P., Castrillón, F., Pedreño, N., \& Moreno-Murcia, A. (2014). Motivación autodeterminada en adultos mayores practicantes de ejercicio físico. Cuadernos de Psicología del Deporte, 14, 149-156.

43. Pérez-López, R., Morales-Sánchez, V., Anguera, M.T., \& Hernández-Mendo, A. (2015). Hacia la calidad de servicio emocional en organizaciones deportivas orientadas a la población infantil: un análisis cualitativo. Revista Iberoamericana de Psicología del Ejercicio y el Deporte, 10, 243-250.

44. Quinn, J., Pascoe, A., Wood, W., \& Neal, D. (2010). Can't control yourself? Monitor those bad habits. Personality and Social Psychology

Bulletin, 36, 499-511. doi: $10.1177 / 0146167209360665$

45. Rabiau, M., Knäuper, B., \& Miquelon, P. (2006). The external quest for optimal balance between maximizing pleasure and minimizing harm: The Compensatory Health Beliefs model. British Journal of Health Psychology, 11, 139-153. doi:10.1348/135910705X52237

46. Radel, R., Pelletier, L., Pjevac,D., \& Cheval,B. (2017). The links between selfdetermined motivations and behavioral automaticity in a variety of real-life behaviors. Motivation and Emotion, 41, 443-454. doi: 10.1007/s11031-017-9618-6

47. Sallis, J., Owen, N., \& Fisher, E. (2015). Ecological models of health behavior. In $\mathrm{K}$. Glanz, B. Rimer, \& K. Viswanath (Eds.), Health behavior and health education: Theory, research, and practice (4th ed.) (pp. 465-485). San Francisco: Jossey-Bass.

48. Salmon, J., Owen, N., Crawford, D., Bauman, A., \& Sallis, J. (2003). Physical activity and sedentary behavior: A population based study of barriers, enjoyment, and preference. Health Psychology, 22, 178-188. doi: 10.1037/02786133.22.2.178

49. Sedentary Behaviour Research Network. (2012). Letter to the editor: Standardized use of the terms "sedentary" and "sedentary behaviours." Applied Physiology, Nutrition, and Metabolism, 37, 540-542. doi:10.1139/h2012-024 


\section{Determinantes psicossociais do comportamento sedentário dos idosos: estudo qualitativo}

50. Stamatakis, E., Davis, M., Stathi, A., \& Hamer, M. (2012). Associations between multiple indicators of objectively-measured and self-reported sedentary behaviour and cardiometabolic risk in older adults. Preventive medicine, 54, 82-87. doi: 10.1016/j.ypmed.2011.10.009

51. Van Cauwenberg, J., Van Holle, V., De Bourdeudhuij, I., Owen, N., \& Deforche, B. (2014). Older adult's reporting of specific sedentary behaviors: validity and reliability. BMC Public Health, 14:734. doi: $10.1186 / 1471-2458-14-734$

52. Van Dyck, D., Cardon, G., \& De Bourdeaudhuij, I. (2017). Which psychological, social and physical environmental characteristics predict changes in physical activity and sedentary behaviors during early retirement? A longitudinal study. PeerJ., 11, 5:e3242. doi: 10.7717/peerj.3242

53. Van Dyck, D., Mertens, L., Cardon, G., De Cocker, K., \& De Bourdeaudhuij, I. (2017). Opinions Toward Physical Activity, Sedentary Behavior, and Interventions to Stimulate Active Living During Early Retirement: A Qualitative Study in Recently Retired Adults. Journal of Aging and Physical Activity, 25, 277-286. doi: 10.1123/japa.20150295

54. Verplanken, B., Walker, I., Davis, A., \& Jurasek, M. (2008). Context change and travel mode choice: Combining the habit discontinuity and self-activation hypotheses.
Journal of Environmental Psychology, 28, 121-127. doi:10.1016/j.jenvp.2007.10.005

55. WHO (2016). Global Strategy and Action Plan on Ageing and Health.

56. Withall, J., Stathi, A., Davis, M., Coulson, J., Thompson, J., \& Fox, K. (2014). Objective indicators of physical activity and sedentary time and associations with subjective wellbeing in adults aged 70 and over. International journal of environmental research and public health, 11, 643-56. doi: 10.3390/ijerph110100643

57. Wood, W. (2017). Habit in Personality and Social Psychology. Personality and social psychology review, 21, 389-403. doi: $10.1177 / 1088868317720362$ 